\title{
Condições e modificações no processo de trabalho: concepções de Agentes
}

\author{
Comunitários de Saúde
}

\author{
Karin Gabriele Bender ${ }^{1}$ \\ Amanda Corrêa dos Santos ${ }^{2}$ \\ Bruno Dittberner Dutra ${ }^{3}$ \\ Luciane Maria Schmidt Alves ${ }^{4}$ \\ Ari Nunes Assunção ${ }^{4}$ \\ Suzane Beatriz Frantz Krug ${ }^{4}$
}

\section{RESUMO}

O objetivo do estudo foi investigar as condições de trabalho do Agente Comunitário de Saúde (ACS) e as modificações necessárias para o aprimoramento das mesmas. Estudo transversal, descritivo do tipo quantitativo-qualitativo, com 251 ACS de 34 equipes de Estratégias de Saúde da Família e de 5 Estratégias de Agentes Comunitários de Saúde dos 13 municípios da 13 ${ }^{\text {a }}$ Coordenadoria Regional de Saúde/RS. Os dados foram coletados com o formulário "Inventário de Trabalho e Risco de Adoecimento" e analisados obtendo-se o escore fatorial dos fatores com o cálculo da média dos itens. Para o estudo qualitativo, cujos dados foram analisados por meio da Análise de Conteúdo, 16 sujeitos participaram de entrevistas, em uma amostragem intencional por município e tipo de estratégia de saúde. Evidenciou-se uma avaliação moderada/crítica no fator condições de trabalho. Os ACS desejam a modificação do ambiente físico do local de trabalho, apontaram a falta de material de apoio e educativo para realização das atividades, de auxílio financeiro e de transporte para as visitas domiciliares. Infere-se que os fatores citados podem ser considerados como dificultadores para a execução do trabalho dos ACS na região estudada.

Palavras-chave: Agentes Comunitários de Saúde. Estratégia Saúde da Família. Saúde do Trabalhador.

\footnotetext{
ABSTRACT

The goal of the study was to investigate the working conditions of the Community Health Workers (ACS) and the necessary modifications to improve them. Crosssectional, exploratory and descriptive study of quantitative-qualitative type with 251 ACS of 34 Family Health Strategies and 5 Community Health Agents Strategies from thirteen municipalities of 13th Regional Health Coordination of Rio Grande do Sul. Data were collected with the form The Work and Illness Risks Inventory (ITRA) and analyzed obtaining the factor score of the factors by calculating the average of the items. For the qualitative study, which data were analyzed using Content Analysis, 16 subjects participated in interviews, in an intentional sampling by municipality and type of health strategy. Data showed a moderate/critical evaluation

${ }^{1}$ Aluna do Curso de Enfermagem da Universidade de Santa Cruz do Sul - UNISC.

${ }^{2}$ Aluna do Curso de Odontologia da Universidade de Santa Cruz do Sul.

${ }^{3}$ Aluno do Curso de Fisioterapia da Universidade de Santa Cruz do Sul.

${ }^{4}$ Professores do Departamento de Enfermagem e Odontologia na Universidade de Santa Cruz do Sul. <skrug@unisc.br>
} 
in working conditions factor. ACS desire the modification of the physical environment of the workplace, they pointed lack of educational supporting material for carrying out the activities and the lack of financial aid or means of transportation to go to the residences of users in home visits. It is inferred that the factors cited can be considered as complicating factors for the ACS work perform in the studied area

Keywords: Community Health Workers. Family Health Strategy. Occupational Health.

\section{INTRODUÇÃO}

O termo trabalho traduz essencialmente uma atividade humana realizada em diferentes situações e condições, podendo contribuir para o bem-estar ou não do trabalhador. Neste sentido, cada categoria profissional está exposta a diferentes situações que podem ser favoráveis ou desfavoráveis para o desenvolvimento das suas atribuições (SOUZA e FREITAS, 2011). Entende-se como condições de trabalho as circunstâncias em que a atividade dos trabalhadores se desenvolve e em que estado eles se encontram para atender as demandas que lhes são apresentadas (ASSUNÇÃO e BELISÁRIO, 2007).

O processo de trabalho do ACS realiza-se, muitas vezes, sob condições não adequadas, o que pode interferir no desempenho de suas atividades. Este profissional tem importante contribuição para a consolidação do Sistema Único de Saúde (SUS), fortalecendo a integração entre os serviços da Atenção Primária à Saúde e a comunidade. Sua atribuição consiste em realizar atividades de prevenção de doenças e promoção da saúde, através de ações domiciliares ou comunitárias, individuais ou coletivas, desenvolvidas conforme as diretrizes do SUS no âmbito da Atenção Básica à Saúde (BRASIL, 2011).

Conforme o Brasil (2012), a Atenção Básica se constitui como "um conjunto de ações de saúde, no âmbito individual e coletivo, que abrangem a promoção e a proteção da saúde, a prevenção de agravos, o diagnóstico, o tratamento, a reabilitação e a manutenção da saúde". Segundo Tomasi et al (2008), embora os trabalhadores da atenção básica constituam um enorme contingente de força de trabalho, no contexto do SUS e do país, pouco se sabe sobre suas condições de trabalho.

O trabalho dos ACS preconiza a execução de suas atribuições nos princípios da atenção integral à saúde e da humanização das práticas. Entretanto, para que tenha um resultado efetivo, há necessidade de serem disponibilizados instrumentos e 
condições de trabalho que sejam compatíveis com a sua função. Por serem considerados o elo de ligação entre a população e a unidade de saúde ficam expostos a situações de trabalho e realidades sociais geradas pela pobreza e desigualdades. Além disso, precisam lidar diariamente com dificuldades e deficiências dos demais níveis do sistema de saúde, demandando o desenvolvimento de habilidades para superar os desafios que podem aparecer no seu cotidiano de trabalho. (BRAGA, CARVALHO, BINDER, 2010; SILVA e DALMASO, 2002)

Segundo Ribeiro, Amaral e Staliano (2015), o profissional que atua na Atenção Básica de Saúde, não raramente, se depara com a escassez de recursos e tecnologias para a resolução dos problemas. Além disso, o campo de trabalho do ACS é permeado pelos tradicionais riscos laborais do campo da saúde (químicos, físicos, ergonômicos e biológicos).

Partindo da premissa que este estudo pode contribuir para evidenciar aspectos do trabalho em saúde, objetivou-se investigar as condições de trabalho de ACS dos 13 municípios da $13^{\text {a }}$ Coordenadoria Regional de Saúde, bem como as modificações necessárias, na percepção dos sujeitos, para o aprimoramento das mesmas.

\section{FUNDAMENTAÇÃO TEÓRICA}

O ACS é um profissional que integra a equipe de Atenção Básica, atuando exclusivamente no âmbito do Sistema Único de Saúde. Por meio de visitas domiciliares este profissional deve desenvolver ações de promoção da saúde, prevenção de doenças e de vigilância à saúde, acompanhando todas as famílias de seu território, mensalmente. O ACS exerce várias atribuições, entre as quais destacase o incentivo à formação de conselhos locais de saúde, educação em saúde, visitas domiciliares, ações básicas de promoção de saúde e prevenção de doenças, cadastro das famílias de sua área e mapeamento de sua área de atuação (COSTA e CARBONE, 2009).

A atenção básica, campo de trabalho do ACS, não é um campo de atenção simplificado e exige do trabalhador um conjunto de tecnologias e atributos para a produção dos serviços de saúde. Estas tecnologias estão divididas em tecnologias duras, leve-duras e leves. As tecnologias duras são os equipamentos e máquinas utilizadas para criação do produto originado do trabalho morto, as leve-duras constituem-se pelos os saberes, normas e protocolos e pelo conhecimento produzido pelas áreas do saber. Já as tecnologias leves compreendem relações de interação e 
subjetividade, e têm como produto a criação de vínculo (MERHY e FEUERWERKER, 2009).

Como o trabalho do ACS tem como base a aproximação com o usuário e a criação de vínculo, é composto majoritariamente pelo uso de tecnologias leves para a produção do cuidado. Esta atividade realizada mediante o encontro do trabalhador com o usuário vincula o trabalhador e o relaciona com seu ato produtivo, bem como com suas relações com os colegas de trabalho e com os usuários de seus produtos, sendo, portanto, um trabalho vivo em ato (MERHY, 2002). De acordo com Jardim e Lancman (2009), o Agente Comunitário de Saúde é o profissional que está em um contato direto com a comunidade, e, por conviver diariamente com ela, tem a função de servir como um elo entre a comunidade e a equipe das Estratégias de Saúde da Família (ESF) e/ou Unidades Básicas de Saúde (UBS). Dessa forma, o ACS promove a integração entre a equipe de saúde e a comunidade adstrita à UBS e/ou ESF, pois é o profissional que conhece a dinâmica social daquela população mais intimamente em decorrência da sua identificação com os moradores do bairro (BRASIL, 2012; MENEGUSSI, OGATA e ROSALINI, 2014).

Segundo Lopes et al (2012) a dinâmica de trabalho dos ACS é complexa e apresenta diversas particularidades, potencializada pelo fato de que estes trabalhadores vivenciam a realidade do bairro onde residem, trabalham e dirigem suas ações de cuidado, e, por ser morador daquela região, vivencia diariamente todas as dificuldades e barreiras que encontra ao adentrar a casa de seus vizinhos (MENEGUSSI, OGATA e ROSALINI, 2014).

Desta maneira, o trabalho do ACS é composto por esses dois mundos, a comunidade e a equipe de saúde, e caracterizado por ser de grande complexidade, haja vista que o mesmo adentra nos domicílios e recebe diariamente as queixas dos moradores, comprometendo-se de forma direta com a necessidade de dar respostas e encaminhamentos aos problemas encontrados. Ao mesmo tempo, ao levar a demanda para a equipe, o ACS pode se deparar com sentimento de impotência, agindo conforme as possibilidades e os limites dela e do próprio sistema de saúde (BANDEIRA, 2014).

Assim, o processo de trabalho do ACS pode ser caracterizado como um trabalho "vivo em ato" através de duas dimensões: uma, é a da atividade como construtora de produtos, de sua realização através da produção de bens, de diferentes tipos, e que está ligada à realização de uma finalidade. Já a outra dimensão vincula- 
se ao produtor do ato, o trabalhador, e sua relação com seu ato produtivo e os produtos que realiza, bem como com suas relações com os outros trabalhadores e com os possíveis usuários de seus produtos (MERHY, 2007).

Outro fator de grande relevância é o ACS ter uma convivência cotidiana perante os problemas encontrados na população e, principalmente, a atuação na criação de vínculos com a comunidade, sem ter treinamento adequado para o enfrentamento dessas dificuldades que surgem no seu trabalho (JUSTO, GOMES e SILVEIRA, 2015; BARALHAS e PEREIRA, 2013).

Por outro lado, durante o período de trabalho, os ACS são expostos a algumas sobrecargas, entre elas estão: horários incertos no momento das visitas domiciliares, dificuldade para cumprir metas, falta de privacidade e liberdade dentro do bairro, carga emocional e acidentes de trabalho no meio do percurso das visitas domiciliares (WAI e CARVALHO, 2009).

Além da carga emocional, os ACS precisam lidar com fatores da organização do trabalho que influenciam nas suas condições laborais e prejudicam o cumprimento de suas funções. Segundo estudos, são alguns destes fatores: o trabalho em equipe; o relacionamento com os gestores das unidades básicas de saúde; o planejamento das atividades; o vínculo com os usuários do serviço; adaptação e dificuldade pra atender e conviver com a comunidade; atendimento de demandas superiores ao preconizado pelo Ministério da Saúde; excesso de burocracia; adversidades no ambiente e na organização do trabalho (WAI e CARVALHO, 2009). Essas fragilidades no serviço, como a carência de meios para a execução das tarefas, diminui a capacidade do trabalhador de responder as adversidades e as demandas da comunidade. Neste sentido, o trabalho em saúde não depende apenas das tecnologias leves, mas também do uso de tecnologias duras (MERHY, 2007).

Quando existe uma falha entre a realidade imposta na organização do trabalho e as expectativas do agente comunitário, ocorre sofrimento, implicando diretamente no enfrentamento das pressões no ambiente de trabalho, causando uma espécie de instabilidade psicológica (MENDES, 1995). O ACS refere ansiedade tanto na relação com a comunidade como com a equipe, especialmente quando se sente pressionado entre ambos, tendo em vista que as dificuldades enfrentadas para o desenvolvimento do exercício profissional enquanto mediadores e tradutores acarretam tanto na produção do cuidado como também no atendimento às demandas de saúde dos moradores (SANTOS, VARGAS e REIS, 2014). 


\section{MATERIAIS E MÉTODOS}

Este estudo trata-se de um recorte da pesquisa "Sofrimento no trabalho de Agentes Comunitários de Saúde: um estudo nos município da $13^{\mathrm{a}}$ Coordenadoria Regional de Saúde - RS” desenvolvida pelo Grupo de Estudos e Pesquisa em Saúde (GEPS) da Universidade de Santa Cruz do Sul (UNISC). O estudo de trajetória transversal, descritivo, do tipo quantitativo-qualitativo teve como integrantes 251 ACS de 34 equipes de Estratégias de Saúde da Família (ESF) e de cinco equipes de Estratégias de Agentes Comunitários de Saúde (EACS) dos 13 municípios de abrangência da $13^{\text {a }}$ Coordenadoria Regional de Saúde (CRS) $-28^{a}$ Região de Saúde do Rio Grande do Sul.

A 28 Região de Saúde, localizada na região dos Vales, é assessorada administrativamente pela $13^{a}$ Coordenadoria Regional de Saúde, sendo composta por 13 municípios e abrangendo uma população de 343.858 habitantes (IBGE, 2014). Esses municípios, em sua maioria, são constituídos por descendentes de alemães e açorianos, dedicados na área rural à criação de suínos, aves ou gado leiteiro, ao cultivo de milho, fumo, eucalipto, frutas, ou ainda, a associação de diversas culturas. $\mathrm{Na}$ área urbana, destacam-se o comércio e inúmeras indústrias, o que confere à região, um expressivo desenvolvimento econômico. Em relação à Atenção Básica, os treze municípios totalizam, atualmente, 46 UBS e 44 ESF.

Para a coleta dos dados foram utilizados dois instrumentos: o formulário "Inventário de Trabalho e Risco de Adoecimento" (ITRA), validado em estudos de Mendes e Ferreira (2007) e uma entrevista composta por 17 questões. O ITRA, é um inventário composto por quatro dimensões (escalas) que avaliam a inter-relação entre trabalho e adoecimento e objetiva investigar os fatores que podem contribuir para o processo de adoecimento no trabalho e seus efeitos sob o trabalhador, além de traçar um perfil das dimensões dessa relação no que se refere ao contexto de trabalho, às exigências, vivências e danos. Para este estudo considerou-se as 10 questões que compõem o fator "Condições de Trabalho" - definido como a qualidade do ambiente físico, posto de trabalho, equipamentos e material disponibilizado para a execução do mesmo - da Escala de Avaliação do Contexto de Trabalho (EACT - escala do tipo Likert de 5 pontos composta por 31 questões e dividida em três fatores: organização do trabalho, condições de trabalho e relações socioprofissionais). Já das questões da entrevista, com o intuito de aprofundar os dados quantitativos obtidos, 
selecionou-se para o presente estudo uma questão norteadora: "Se fosse possível mudar algo no seu trabalho, o que você sugere para mudança? Por quê?”.

O primeiro instrumento foi aplicado aos 251 ACS e o segundo a 16 sujeitos que participaram de entrevistas, em uma amostragem intencional por município e tipo de estratégia de saúde. Estes dados foram coletados em registros eletrônicos, seguindo um roteiro de questões abertas em que os sujeitos responderam expressando-se livremente de acordo com o estímulo recebido. Os entrevistados foram identificados como ACS 1; ACS 2; ACS 3 e assim sucessivamente até o último sujeito, para garantir o anonimato.

Os dados quantitativos foram organizados com o auxílio do Software Statistical Package for Social Sciences 20.0 (SPSS). Na análise dos dados do fator "Condições de trabalho" obteve-se o escore fatorial com o cálculo da média entre os itens, considerando-se o ponto médio e o desvio padrão. Estes resultados foram classificados em graves (escore acima de 3,7), moderados/críticos (escore de 2,3 a 3,69) e satisfatórios (escore abaixo de 2,29). Já os dados qualitativos, obtidos por meio das entrevistas, foram transcritos, organizados e analisados na perspectiva de Análise de Conteúdo segundo Bardin (2010). A pesquisa foi aprovada pelo Comitê de Ética em Pesquisa da Universidade de Santa Cruz do Sul - UNISC sob o protocolo 713.475 .

\section{RESULTADOS E DISCUSSÃO}

Os resultados demonstram que há complementariedade entre os dados quantitativos e qualitativos, à medida que o desejo de mudança exposto nas entrevistas demonstra a fragilidade das condições de trabalho citada pelos ACS de cada região estudada.

Os ACS participantes do estudo eram predominantemente do sexo feminino $(92,8 \%)$, com idades entre 31 a 40 anos $(37,5 \%)$, casados $(48,6 \%)$ e com 1 a 2 filhos $(64,9 \%)$. Quanto à escolaridade, 54,2\% tem ensino médio completo e 13,2\% estão cursando ou já concluíram o ensino superior. A maioria atua em ESFs $(59,8 \%)$ e, o restante, em EACS (40,2\%), predominantemente na área urbana (50,2\%), seguido da área rural $(43,8 \%)$ e em ambas as áreas $(5,25 \%)$.

Visto que o trabalho do ACS está muito ligado a formação de vínculo e ao cuidado, o predomínio do sexo feminino pode estar relacionada a uma divisão social e sexual do trabalho que associa a mulher ao papel de cuidadora e a imagem do 
feminino a uma ideia de vocação ao cuidado do outro (BARBOSA, 2012). Verificase, ainda, que esta modalidade de trabalho "vocacional" ocasiona a exploração da trabalhadora, frequentemente alocando-a a setores mais explorados, mal remunerados e precarizados, o que repercute negativamente em sua saúde (SANTOS, 2010). No caso destas ACS, além da jornada de trabalho nos serviços de saúde, pode haver uma dupla ou tripla jornada de trabalho, haja vista que essa divisão sexual do trabalho e ideologia de gênero também associa o feminino ao cuidado materno, implicando em acúmulo de tarefas sobre estas trabalhadoras, que devem conciliar o trabalho assalariado com o cuidado com os filhos e o lar (BARBOSA, 2012).

O resultado da análise quantitativa evidenciou um escore de 2,54 pontos avaliação considerada como moderada/crítica no fator condições de trabalho. Itens como "o ambiente físico é desconfortável”, "as condições de trabalho são precárias", "existe muito barulho no ambiente de trabalho" compõe este fator. A Tabela 1 apresenta a média, o desvio-padrão e a avaliação do fator no estudo realizado.

\begin{tabular}{lcc}
\hline \multicolumn{1}{c}{ EACT } & Média \pm DP* & Avaliação \\
\hline \multicolumn{1}{c}{ Fator: Condições de Trabalho } & $2,54 \pm 0,83$ & Moderada/Crítica \\
\hline $\begin{array}{l}\text { Item 22 - As condições de trabalho são } \\
\text { precárias }\end{array}$ & $2,81 \pm 1,18$ & Moderada/Crítica \\
Item 23 - O ambiente físico é & $2,58 \pm 1,23$ & Moderada/Crítica \\
desconfortável & & \\
Item 24 - Existe muito barulho no & $2,37 \pm 1,13$ & Moderada/Crítica \\
ambiente de trabalho & & \\
\hline
\end{tabular}

*DP=Desvio Padrão

Segundo Mendes e Ferreira (2007), as condições de trabalho são definidas como "qualidade do ambiente físico, posto de trabalho, equipamentos e materiais disponibilizados para a execução do trabalho". Já segundo Assunção e Belisário (2007), as condições de trabalho são fatores, cujos efeitos, articulados a outros, determinam tanto a saúde dos sujeitos que operam os sistemas quanto os meios que eles dispõem para o exercício pleno de suas capacidades.

Um escore de avaliação considerado como moderado - crítico pode demonstrar que as condições de trabalho nas quais o ACS realiza as suas atribuições 
não correspondem ao ideal, ou ainda, não atendem às expectativas destes trabalhadores, o que pode estar comprometendo o seu processo de trabalho. Segundo Reinhardt e Fischer (2009), as condições de trabalho quando não adequadas ao trabalhador, oferecem risco à saúde e não favorecem a capacidade para o trabalho e a produtividade.

Dejours (1987) afirma que as condições externas oferecidas no ambiente de trabalho que não atendam à satisfação dos desejos do trabalhador prejudicam sua saúde. Neste sentido, o trabalhador que exerce suas atividades em ambiente adverso, com condições de trabalho não adequadas, está sujeito a exercer suas atribuições dentro de um padrão que não corresponde às expectativas destes sujeitos, podendo vivenciar sentimentos de sofrimento relacionado ao trabalho.

No que se refere a análise das entrevistas, destaca-se a categoria temática "sugestões de mudança" com as citações dos ACS que desejam modificar aspectos referentes ao ambiente físico de seu local de trabalho. Entre os aspectos pontuados pelos ACS, ressalta-se como fator que dificulta o desempenho de suas atividades a falta de auxilio ou subsídios para o deslocamento dentro da comunidade, uma vez que para suprir estas necessidades e poder realizar seu trabalho, os próprios ACS arcam com estas despesas, conforme exposto na seguinte fala:

"Eu acho que eles podiam ajudar na gasolina, pra uns eles deram bicicleta, mas não tem como trabalhar de bicicleta... eu nem ganhei bicicleta. Aí eu tenho a moto, gasta pneu, gasta gasolina e eles não ajudam com nada, tu tem que ir por conta própria; é muito longe...no meu caso agora, só tem ônibus escolar, não pode viajar mais aí tu tem que se virar por conta própria, se tu tem condição de trabalhar tu vai, se não tu fica em casa, porque eles não ajudam em nada." (ACS 1)

Conforme preconizado pelo Ministério da Saúde todas as famílias e pessoas do território de cada ACS devem ser acompanhadas por meio da visita domiciliar, através do qual se desenvolvem as ações em saúde. Sua atuação não está restrita ao domicílio, ocorrendo também nos diversos espaços comunitários e na Unidade de Saúde a qual está vinculado, o que exige que o trabalhador se desloque até estes locais (BRASIL, 1999).

Condições de trabalho inadequadas ou insuficientes afetam os trabalhadores, os serviços nos quais se inserem e também a relação entre estas duas esferas, dificultando o desenvolvimento do SUS e a qualidade dos serviços essenciais prestados (CAVALCANTE e LIMA, 2013). O fato do ACS desenvolver seu trabalho 
dentro da estrutura física da unidade e nos domicílios ou espaços públicos, onde está sujeito às mudanças climáticas, intempéries e situações de violência, calor, frio, insolação excessiva, entre outros eventos inconvenientes, configura seu cenário de prática como um ambiente adverso, dificultando e interferindo no bom desenvolvimento de suas atividades. Em um estudo realizado por Souza e Freitas (2011), os ACS consideraram uma forma de violência institucional o não oferecimento de infraestrutura para o trabalho, e entendem que melhores condições de trabalho significariam melhor assistência à saúde da população. Os ACS entrevistados sentem-se desamparados pela unidade e esta situação, além de prejudicar suas ações para com a comunidade, pode predispor esse trabalhador a sensações de sofrimento e levar ao adoecimento.

Além destas dificuldades, os sujeitos citaram a falta de material de apoio para realização das ações em saúde. Através da fala do ACS é possível perceber que há vontade de realizar as ações, mas que nem sempre são disponibilizados materiais e condições de trabalho compatíveis com o que é necessário realizar:

A gente tem a vontade, tem as ideias, mas não tem o material, a base pra poder desempenhar melhor. A gente ter mais condições mesmo de trabalho... a gente precisar de materiais, por exemplo, educativo, tu dizer que precisa um material pra trabalhar com a comunidade e tu ter esse material, e a gente não tem... (ACS 2)

[...] essas pastas aqui, foi a gente que comprou, caneta a gente tá comprando, protetor solar? Se eu quiser, se eu me preocupar comigo, eu vou lá e compro com o meu salário... (ACS 3)

Esses trabalhadores de saúde deixaram transparecer em suas falas que o processo de trabalho tem sido realizado sob precárias condições materiais, o que pode prejudicar sua motivação para o trabalho e ameaçar a qualidade prestada à população, resultados estes que estão em consonância com o estudo de Souza e Freitas (2011).

Esta situação, entretanto, não é exclusividade da região estudada. Em estudo realizado sobre as condições de trabalho das equipes de ESF de um município do estado do Rio Grande do Norte, os autores também encontraram como fatores que dificultam o trabalho a insuficiência de materiais para a execução de suas atividades, como equipamentos, instrumentos, espaço físico além de divergências de salario dentre os profissionais da equipe (PINTO, MENEZES e VILLA, 2010). Um outro estudo realizado na realidade de Maceió reforça as ponderações anteriores ao 
demonstrar que há insuficiência de espaço físico, insumos materiais para o desenvolvimento das atividades (CAVALCANTE e LIMA, 2013).

Os relatos demonstram também que há uma distância considerável entre o trabalho imaginado e trabalho produzido (RONCHI, 2010), uma vez que o profissional cria uma expectativa em relação a sua competência no sistema de saúde, ao mesmo tempo que as condições de trabalho não respondem a esta necessidade (SOUZA e FREITAS, 2011). O ACS demonstra vontade de realizar as suas tarefas de maneira satisfatória, entretanto as condições de trabalho podem não estar permitindo que ele desenvolva a sua potencialidade, ou seja, pode estar restringindo a margem de ação deste trabalhador. Conforme Dejours (1996), o trabalho que não propicia ao trabalhador a possibilidade de criação e escolha do modo como será realizada a tarefa, pode ser prejudicial a sua saúde. O trabalho se apresenta ao sujeito como parte de seu mundo externo, de seu próprio corpo e de suas relações sociais, podendo ser fonte de prazer ou sofrimento a partir do momento em que as condições por ele oferecidas atendam ou não aos seus desejos inconscientes (MENDES, 1995).

O trabalho é considerado como atributo humano, pois só o homem consegue idealizar o resultado final. Para a realização do trabalho, além do esforço, é necessário vontade durante todo o curso do seu desenvolvimento. Neste sentido, quaisquer fatores que atuem sobre algum dos elementos que compõe este processo tendem a modificá-lo de forma positiva ou negativa (SOUZA e FREITAS, 2011).

\section{CONCLUSÃO}

O estudo demonstrou que os ACS da $13^{\text {a }}$ Coordenadoria Regional de Saúde do Rio Grande do Sul estão exercendo suas atividades sob condições de trabalho não consideradas ideais. Neste sentido, identifica-se um contexto de trabalho que pode prejudicar o desenvolvimento das potencialidades destes trabalhadores, podendo ser propicio a situações geradoras de sofrimento. A insuficiência de materiais essenciais, a falta de EPI e de subsídio para o deslocamento até as residências foram pontuados como fatores da organização do trabalho que prejudicam o desempenho das atividades e que levam os trabalhadores a usar outros artifícios para suprir estas necessidades.

O fato dos entrevistados sugerirem possíveis modificações em suas condições de trabalho demonstra que a forma como esse trabalho está configurado desperta a insatisfação e motiva o desejo de mudança. Este sentimento de mudança se efetiva 
no momento em que o ACS atua sobre sua realidade de trabalho e a transforma, buscando, deste modo, compensar a diferença entre a realidade imposta pela organização do trabalho e os anseios do trabalhador.

Este estudo aponta a necessidade de ações com vistas a beneficiar a prática de trabalho do ACS e dos demais integrantes da equipe de saúde, uma vez que os fatores citados são importantes e necessários para uma boa prática assistencial.

\section{REFERÊNCIAS}

ASSUNÇÃO, A. A.; BELISÁRIO, S. A. Condições de trabalho e saúde dos trabalhadores da saúde. Belo Horizonte: Nescon - Núcleo de Educação em Saúde Coletiva, 2007.

BANDEIRA, A. G. Competências do Agente Comunitário de Saúde: subsídio para a avaliação formativa na Estratégia de Saúde da Família. 122 f. 2014. Dissertação (Programa de Pós-Graduação em Enfermagem-Mestrado) - Universidade Federal do Rio Grande do Sul, Porto Alegre, 2014.

BARALHAS, M.; PEREIRA, M. A. O. Prática diária dos agentes comunitários de saúde: dificuldades e limitações da assistência. Revista Brasileira de Enfermagem, Brasília, n. 3, v. 66, p. 358-365, maio/jun.2013.

BARBOSA, R. H. S. et al. Gênero e trabalho em Saúde: um olhar crítico sobre o trabalho de Agentes Comunitárias/os de Saúde. Interface - comunicação, saúde e educação [online]. Botucatu, n. 42, v. 16, p. 751-65. jul./set.2012.

BARDIN, L. Análise de Conteúdo. Lisboa: Edições 70. 2010.

BRAGA，L. C. de; CARVAlHO, L. R. de; BINDER, M. C. P. Condições de trabalho e transtornos mentais comuns em trabalhadores da rede básica de saúde de Botucatu (SP). Ciênc. saúde coletiva, Rio de Janeiro, v. 15, supl. 1, p. 1585-1596, jun.2010.

BRASIL, Ministério da Saúde [MS]. Política Nacional de Atenção Básica. Brasília, DF: Ministério da Saúde. 2012.

BRASIL. PORTARIA No 2.488, DE 21 DE OUTUBRO DE 2011. Aprova a Política Nacional de Atenção Básica, estabelecendo a revisão de diretrizes e normas para a organização da Atenção Básica, para a Estratégia Saúde da Família (ESF) e o Programa de Agentes Comunitários de Saúde (PACS).

BRASIL. Ministério da Saúde. Saúde da família no Brasil: linhas estratégicas para o quadriênio 1999/2002. Brasília: Ministério da Saúde, 1999. 
CAVAlCANTE, M. V. da S.; LIMA, T. C. S. de. A precarização do trabalho na atenção básica em saúde: relato de experiência. Argumentum, Vitória (ES), n.1, v. 5, p. 235-256, jan./jun. 2013.

COSTA, E. M. A.; CARBONE, M. H. Saúde da família: uma abordagem multidisciplinar. 2. ed. Rio de Janeiro: Rubio, 2009.

DEJOURS, C. A Loucura do Trabalho: Estudo de Psicopatologia do Trabalho. São Paulo: Cortez, 1987.

DEJOURS, C. Uma nova visão do sofrimento humano nas organizações. In: CHANLAT, J.-F. (Org.). O indivíduo na organização: dimensões esquecidas. São Paulo: Atlas, 1996.

IBGE. Diretoria de População e Indicadores Sociais. Estimativas da população residente com data de referência $1^{\circ}$ de julho de 2014. Diário Oficial da União, 28 ago. 2014. Brasília, 2014. Disponível em: < http://www.ibge.gov.br/home/estatistica/populacao/estimativa2014/estimativa_dou.s htm>. Acesso em 05 jul. 2015.

JARDIM, T. de A.; LANCMAN, S. Aspectos subjetivos do morar e trabalhar na mesma comunidade: a realidade vivenciada pelo agente comunitário de saúde. Interface - Comunicação, Saúde e Educação, Botucatu, n. 28, v. 13, p. 123135, mar.2009.

JUSTO, C. M. P.; GOMES, M. H. A.; SILVEIRA, C. Limites e imposições dos instrumentos de controle do trabalho de agentes comunitários de saúde da Estratégia Saúde da Família. Saúde e Sociedade, São Paulo, n. 2, v. 24, p. 594-606, 2015.

LOPES, D. M. Q. et al. Agentes Comunitários de Saúde e as vivências de prazer sofrimento no trabalho: estudo qualitativo. Revista Escola de Enfermagem da USP, São Paulo, n. 3, v. 46, p. 633-40, 2012.

MENDES, A. M. B. Aspectos psicodinâmicos da relação homem-trabalho: as contribuições de C. Dejours. Psicol. cienc. prof., Brasília, n. 1-3, v. 15, p. 3438, 1995.

MENDES, A. M. B.; FERREIRA, M. C. Inventário sobre Trabalho e Riscos de Adoecimento - ITRA: instrumento auxiliar de diagnóstico de indicadores críticos no trabalho. In: MENDES, A. M. B. (org.) Psicodinâmica do trabalho: teoria, método e pesquisa. São Paulo: Casa do Psicólogo, p. 111-126, 2007.

MERHY, E. E. A reestruturação produtiva na saúde, a produção do cuidado e a cartografia do trabalho vivo em ato. São Paulo: HUCITEC, 2002.

MERHY, E. E. Saúde: a cartografia do trabalho vivo. 3. ed. São Paulo: Editora Hucitec, 2007.

MERHY, E. E.; FEUERWERKER, L.C.M. Novo olhar sobre as tecnologias de saúde: uma necessidade contemporânea. In: MANDARINO, A.C.S.; GOMBERG, E. 
(Orgs.). Leituras de novas tecnologias e saúde. São Cristóvão: Editora UFS, 2009. p.29-74.

MENEGUSSI, J. M.; OGATA, M. N.; ROSALINI, M. H. P. O agente comunitário de saúde como morador, trabalhador e usuário em São Carlos, São Paulo. Trabalho, Educação e Saúde. n.1, v. 12, p. 87-106, 2014.

PINTO, E. S. G.; MENEZES, R. M. P. de; VILLA, T. C. S. Situação de trabalho dos profissionais da Estratégia Saúde da Família em Ceará-Mirim. Revista da Escola de Enfermagem da USP, São Paulo, n. 3, v. 44, p. 657-664, set. 2010.

REINHARDT, E. L.; FISCHER, F. M. Barreiras às intervenções relacionadas à saúde do trabalhador do setor saúde no Brasil. Rev. Panam Salud Publica, Washington, n. 5, v. 25, p. 411-417, maio. 2009.

RIBEIRO, S. F. R.; AMARAL, P. J. V. do; STALIANO, P. Sofrimento psíquico do Agente Comunitário de Saúde: revisão bibliográfica. R. Laborativa, n. 1, v. 4, p. 7996, abr. 2015.

RONCHI, C. C. Sentido do Trabalho: saúde e qualidade de vida. Curitiba: Juruá, 2010.

SANTOS, M. M. Igualdade nas relações de gênero na sociedade do capital: limites, contradições e avanços. Katálysis, n. 1, v.13, p.11-9, 2010

SANTOS, I. E. R.; VARGAS, M. M.; REIS, F. P. Estressores laborais em agentes comunitários de saúde. Revista Psicologia: Organização e Trabalho, n.3, v.14, p. 324-335, 2014.

SILVA, J. A. D.; DALMASO, A. S. W. O agente comunitário de saúde e suas atribuições: os desafios para os processos de formação de recursos humanos em saúde. Interface - Saúde, Educação, Comunicação, Botucatu, n. 10, v. 6, p. 7583, fev. 2002.

SOUZA, L. J. R. de; FREITAS, M. do C. S de. O agente comunitário de saúde: violência e sofrimento no trabalho a céu aberto. Revista Baiana de Saúde Pública, Salvador, n. 1, v. 35, p. 96-109, 2011.

TOMASI, E. et al. Perfil sócio-demográfico e epidemiológico dos trabalhadores da atenção básica à saúde nas regiões Sul e Nordeste do Brasil. Caderno de Saúde Pública, Rio de Janeiro, v. 24, supl. 1, 2008.

WAI, M. F. P.; CARVALHO, A. M. P. O trabalho do Agente Comunitário de Saúde: fatores de sobrecarga e estratégias de enfrentamento. Revista Enfermagem UERJ, Rio de Janeiro, n. 4, v. 17, pp. 563-568, 2009. 


\section{Como citar este documento:}

BENDER, Karin Gabriele et al. Condições e modificações no processo de trabalho: concepções de Agentes Comunitários de Saúde. Revista Jovens Pesquisadores, Santa Cruz do Sul, v. 6, n. 2, nov. 2016. ISSN 2237-048X. Disponível em: $<$ https://online.unisc.br/seer/index.php/jovenspesquisadores/article/view/7283>. Acesso em: ... doi:http://dx.doi.org/10.17058/rjp.v6i2.7283. 